\title{
Enhanced Sensing Performance of Integrated Gas Sensor Devices ${ }^{+}$
}

\author{
Ayoub Lahlalia ${ }^{1, *}$, Olivier Le Neel ${ }^{2}$, Ravi Shankar ${ }^{2}$, Siegfried Selberherr ${ }^{1}$ and Lado Filipovic ${ }^{1}$ \\ 1 Institute for Microelectronics, TU Wien, 1040 Vienna, Austria; selberherr@iue.tuwien.ac.at (S.S.); \\ filipovic@iue.tuwien.ac.at (L.F.) \\ 2 STMicroelectronics Pte Ltd., Singapore 569508, Singapore; olivier.leneel@st.com (O.L.N.); \\ ravi.shankar@st.com (R.S.) \\ * Correspondence: lahlalia@iue.tuwien.ac.at; Tel.: +43-(1)5880136015 \\ + Presented at the Eurosensors 2018 Conference, Graz, Austria, 9-12 September 2018.
}

Published: 7 December 2018

\begin{abstract}
Semiconducting metal oxide (SMO) gas sensors, dedicated to wearable devices were designed, fabricated, and characterized in terms of power consumption, thermal distribution, and sensing capability. The sensors demonstrate a sensitivity down to ppb-level VOC concentrations at a low power consumption of $10.5 \mathrm{~mW}$. To further enhance the baseline stability and sensing response characteristics at low power consumption, a new sensor structure is proposed. The design implements novel aspects in terms of fabrication and microheater geometry, leading to improved sensor performance which enables new applications for SMO gas sensors. In this work, two designs were analyzed using experimental characterization and simulation. The results of the analyses of the two sensors are comparatively reported.
\end{abstract}

Keywords: semiconducting metal oxide; gas sensor; microheater; electo-thermal simulation

\section{Introduction}

Recently, the desire for SMO gas sensors suitable for portable devices such as smartphones and smartwatches has been particularly strong. This type of gas sensor shows potential compared to other sensors, especially in terms of fabrication cost, footprint, sensitivity, and response time [1]. Nevertheless, a further decrease in the power consumption and enhanced selectivity as well as reliability are still necessary in order to enable practical integration into portable devices. The aim of this work is to further enhance the sensitivity towards Volatile Organic Compounds (VOCs) and to improve the baseline stability at low power consumption, while making the sensor compatible with Application Specific Integrated Circuit (ASIC) features. For this purpose, a novel design was conceived to optimize the temperature uniformity over the active area, while reducing the overall resistance of the microheater.

\section{Materials and Methods}

The microheater is one of the key components of the SMO gas sensor as it defines several aspects of the sensor performance, such as power consumption, sensitivity, and selectivity [2]. To heat the microheater to elevated temperatures, as required for SMO sensing, joule heating is utilized due to its simplicity in implementation. In this study, tantalum-aluminum (TaAl) has been chosen for the microheater material due to its ability to retain its mechanical strength at high operating temperatures and for its low temperature coefficient of resistance (TCR), which minimizes the effects of hotspots [3]. To further improve the sensitivity down to ppb-levels, the sensitive layer must be as large as possible, since the chemical reactions involved are directly linked to the amount of surface area in 
contact with the environment [4]. However, due to the high temperature requirement, a large area means increased power dissipation. The power consumption depends on the microheater resistance and gives rise to a compromise between a large heated area and a small resistance to reduce the power, as shown in Figure 1. The novel composite heater design presented here allows to heat a large surface at a uniform temperature while having a small resistance, which determines the power consumption. In addition, a small heater enables a very fast thermal response time, allowing the sensor to operate in short-pulsing mode and with a fast response time. Using this new microheater structure, the heater resistance is reduced, while the heated area is considerably increased, which improves the gas sensor sensitivity without a significant increase in power.

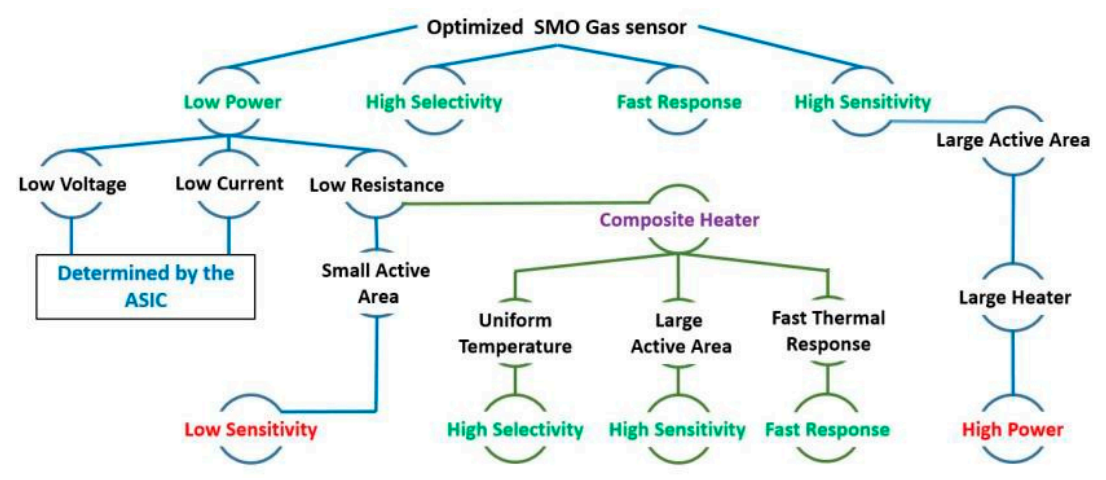

Figure 1. Limitations and required elements to enhance the sensing capability and performance of SMO gas sensors.

The composite heater was fabricated by etching openings in the $\mathrm{AlCu}$ conductive pads of an existing design, the so-called D02 design (Figure 2a) published previously [5]. With the openings, we create a succession of resistances, which are able to concentrate the heating of the sensitive material to desired locations. In the process flow, this etching step appears after the deposition of the TaAl microheater and the $\mathrm{AlCu}$ conductive pads, as shown in Figure $2 \mathrm{~b}$. The total resistance of the heater must be between $50 \Omega$ and $150 \Omega$ in order to match with the ASIC's features.

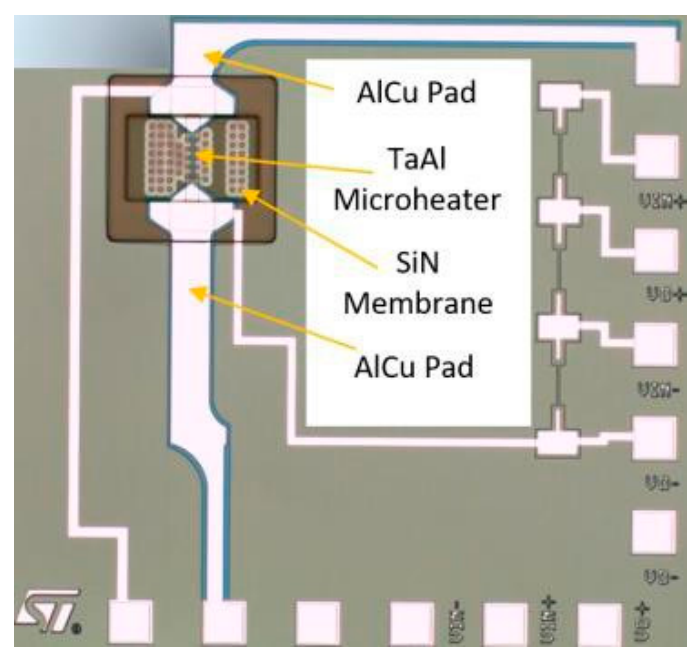

(a)

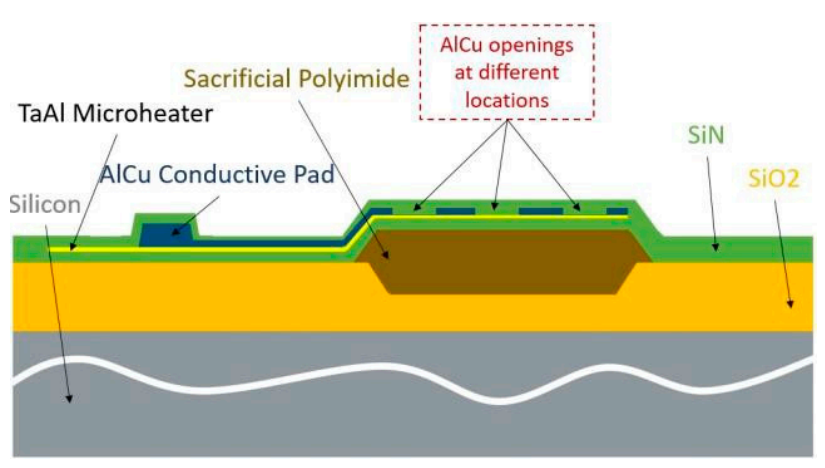

(b)

Figure 2. Structure of the SMO gas sensors. (a) Top view of the initial D02 design. (b) Cross section of the novel composite heater design with the AlCu openings. To form the gas sensor membrane, the sacrificial polyimide was etched as the last step in the fabrication sequence using selective plasma etching in order to achieve low power consumption and good thermal isolation. 


\section{Results}

The Pt doped $\mathrm{SnO}_{2}$ thin films were deposited on two different micro-hotplates in order to assess the most efficient hotplate in terms of power consumption and temperature distribution. Thereafter, sensing characteristics towards formaldehyde at different concentrations were investigated.

\subsection{Device Simulation}

The sensors were designed and simulated using the COMSOL finite element software, where the boundary conditions and initial material temperatures were set to $25^{\circ} \mathrm{C}$ for all materials of the gas sensors and the ambient. The tantalum-aluminum TCR and the temperature of the substrate bottom were fixed at $-100 \mathrm{ppm} /{ }^{\circ} \mathrm{C}$ and $25^{\circ} \mathrm{C}$, respectively. To calculate the heat losses to the air by conduction and convection, $233.46 \mathrm{Wm}^{-2} \mathrm{~K}^{-1}$ was used as the heat transfer coefficient, when the temperature of the top and bottom surface (heated area) of the membrane reaches $300{ }^{\circ} \mathrm{C}[5]$.

Figure $3 \mathrm{~b}$ shows the temperature distribution over the composite heater design, where a high uniformity in temperature distribution over the active sensitive region can be observed. This is a significant improvement to the D02 design as shown in Figure 3a. The improved uniformity in temperature has been achieved in the composite heater design thanks to the high thermal conductivity of the AlCu conductive pads and the SiN membrane and insulation layer. The heating process is no longer activated by the microheater alone, but also by the $\mathrm{AlCu}$ and SiN. Therefore, even if the total resistance is reduced, a larger overall area is achieved by separating the heating locations.

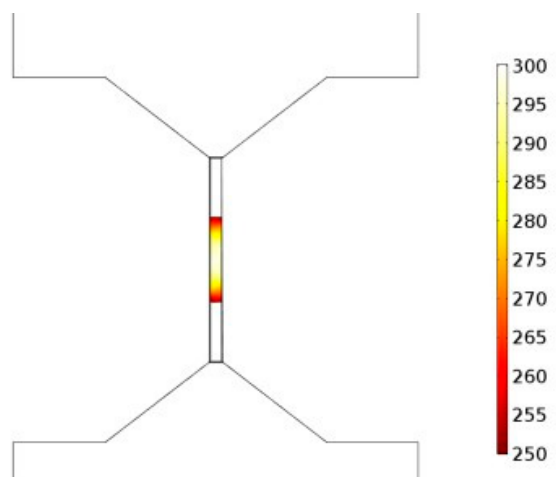

(a)

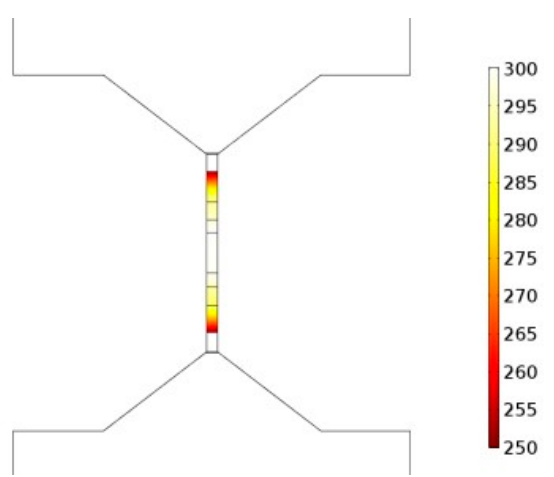

(b)

Figure 3. Temperature distribution $\left[{ }^{\circ} \mathrm{C}\right]$ over the active area of (a) the initial D02 design and (b) the novel composite heater design.

\subsection{Sensing Performance}

Both sensors have been characterized in a micro-chamber with the aim to test and study their performances under formaldehyde exposures. The relative humidity and the gas flow rate were fixed at $55 \%$ and $300 \mathrm{sccm}$, respectively. The sensors were exposed to an increasing formaldehyde concentrations for a duration of $1 \mathrm{~min}$ from $100 \mathrm{ppb}$ to $5 \mathrm{ppm}$. To control the surrounding temperature and humidity, the micro-chamber is placed in an environmental chamber. The gas concentrations inside the micro-chamber were controlled and monitored using several valves, connected to a PC. In order to measure the resistance of the thin film sensitive layers, Keithley 2450 SourceMeters were used at a bias current of $1 \mu \mathrm{A}$.

Figure 4a shows the sensor responses of the composite heater design and the D02 design towards formaldehyde at different concentrations. Each sensor is operating at $10.5 \mathrm{~mW}$ to warm up the layer to a high temperature. An improvement in the sensitivity of the Pt-doped $\mathrm{SnO}_{2}$ thin film towards VOCs has been achieved by using the composite heater instead of the D02 design. The stability of the baseline was also tested for an extended period as shown in Figure $4 \mathrm{~b}$. The composite heater design shows a highly stable baseline compared to the baseline of D02, which shows a drift even after 25 min of operation. 


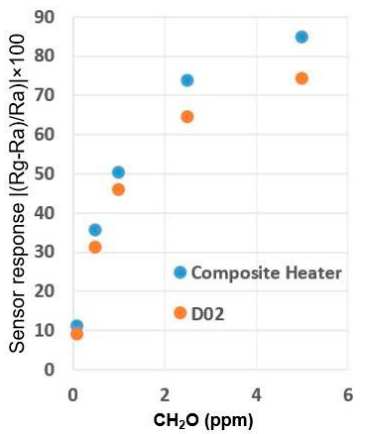

(a)

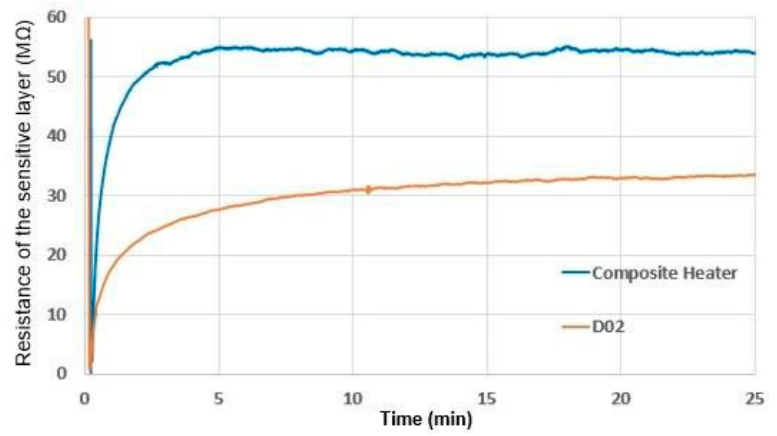

(b)

Figure 4. Sensing characteristics of the composite heater and the D02 design operating at $10.5 \mathrm{~mW}$.

(a) The gas sensor response towards formaldehyde gas at concentrations in the ppb and ppm range.

(b) Baseline response of both sensors during operation for $25 \mathrm{~min}$.

Figure 5 shows the response time and recovery time of the composite heater design when exposed to $1 \mathrm{ppm}$ of formaldehyde. The response time is in the range of 18-20 s, which is very satisfactory for applications dedicated to air quality monitoring. It is defined as the time required for the conductance to change to $90 \%$ of its final value. The recovery time is about $2.3 \mathrm{~min}$.

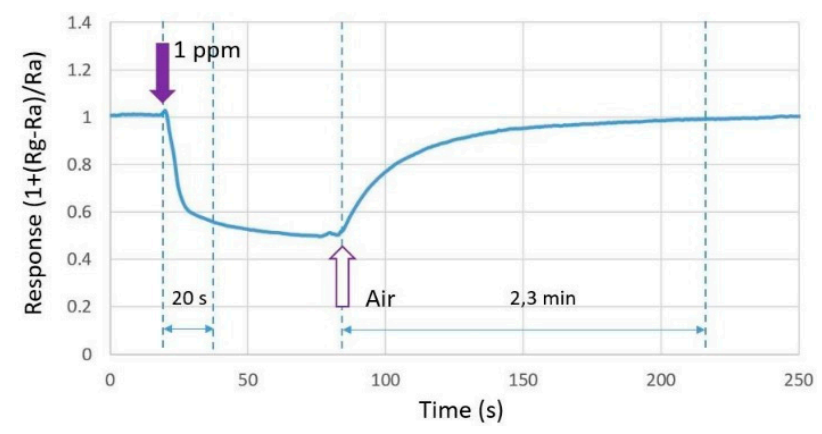

Figure 5. Response time and recovery time of the composite heater design to 1 ppm of formaldehyde.

\section{Discussion}

In order to optimize and improve the performance of the previous design D02, while making the sensor compatible with ASIC features, a novel sensor was designed, fabricated, and characterized. The characteristics of the two designs in terms of sensing capability and thermal behavior have been qualitatively compared. The response of the composite heater towards formaldehyde was found to be significantly improved compared to the $\mathrm{D} 02$ design at $10.5 \mathrm{~mW}$ power consumption. The baseline responses have also been tested for a period of $25 \mathrm{~min}$. The composite heater shows a highly stable baseline compared to that of the D02 design. Concerning the response and recovery times of the sensors, both designs demonstrate satisfactory results.

Author Contributions: Fabrication and characterization was performed at ST Microelectronics, while the simulations were performed at the Institute for Microelectronics, TU Wien. All authors contributed equally to the manuscript.

Acknowledgments: The work has been supported by R\&D at STMicroelectronics Pte Ltd. Ang Mo Kio, Singapore, which has performed the sensor fabrication and characterization.

Conflicts of Interest: The authors declare no conflict of interest. 


\section{References}

1. Yi, S.; Tian, S.; Zeng, D.; Xua, K.; Peng, X.; Wang, H.; Zhang, S.; Xie, C. A novel approach to fabricate metal oxide nanowire-like networks based coplanar gas sensors array for enhanced selectivity. Sens. Actuators B Chem. 2014, 204, 351-559.

2. Mohsen, G.; Ansari, M. Tin Oxide Microheater for Chemical Sensors. IOP Conf. Ser. Mater. Sci. Eng. 2016, 108, doi:10.1088/1757-899X/108/1/012018.

3. Bhattacharyya, P. Technological journey towards reliable microheater development for MEMS gas sensors: A review. IEEE Trans. Device Mater. Reliab. 2014, 14, 589-599.

4. Sharma, A.; Tomar, M.; Gupta, V. Enhanced response characteristics of $\mathrm{SnO}_{2}$ thin film based $\mathrm{NO}_{2}$ gas sensor integrated with nanoscaled metal oxide clusters. Sens. Actuators B Chem. 2013, 181, 735-742.

5. Lahlalia, A.; le Neel, O.; Shankar, R.; Kam, S.Y.; Filipovic, L. Electro-Thermal Simulation \& Characterization of a Microheater for SMO Gas Sensors. J. Microelectromech. Syst. 2018, 27, 529-537.

(C) 2018 by the authors. Licensee MDPI, Basel, Switzerland. This article is an open access article distributed under the terms and conditions of the Creative Commons Attribution (CC BY) license (http://creativecommons.org/licenses/by/4.0/). 